\title{
Combination therapies involving checkpoint-inhibitors for treatment of urothelial carcinoma: a narrative review
}

\author{
Gerald B. Schulz ${ }^{1,2}$, Peter C. Black ${ }^{1}$ \\ ${ }^{1}$ Vancouver Prostate Centre, University of British Columbia, Vancouver, Canada; ${ }^{2}$ Department of Urology, Ludwig-Maximilians-University, Munich, \\ Germany \\ Contributions: (I) Conception and design: Both authors; (II) Administrative support: PC Black; (III) Provision of study materials or patients: None; (IV) \\ Collection and assembly of data: Both authors; (V) Data analysis and interpretation: Both authors; (VI) Manuscript writing: Both authors; (VII) Final \\ approval of manuscript: Both authors. \\ Correspondence to: Dr. Peter C. Black. Vancouver Prostate Centre, University of British Columbia, Level 6, 2775 Laurel St, Vancouver, BC V5Z 1M9, \\ Canada. Email: pblack@mail.ubc.ca.
}

\begin{abstract}
The implementation of immune checkpoint-inhibitors (CPI) has significantly improved the prognosis of a subgroup of patients with urothelial bladder cancer (BC). Still, the majority of patients will progress or experience a recurrence on CPI monotherapy. The next generation of clinical trials is now testing combination therapy with CPI and other agents that target different oncogenic mechanisms in an effort to improve efficacy. The beneficial toxicity profile of CPI but also the approval of CPI combinations in other cancer sites justifies their investigation also in BC. Here we report on clinical trials in muscle-invasive, locally advanced and metastatic BC combining CPI with other therapies, with a focus on the latest results presented at ASCO GU 2020, ASCO 2020 and ESMO 2019 as well as Phase-III trials currently ongoing. Multiple phase I-III clinical trials are investigating the combination of a CPI with a second CPI, with chemotherapy, or with targeted therapies like fibroblast growth factor receptor (FGFR) inhibitors or Nectin-4 inhibitors in different disease states. The results of more than 10 phase-III trials in advanced BC are eagerly awaited. Preliminary data are contradictory, as some trials released promising interim results, while others reported failure to achieve the primary endpoints. Taken together, combining CPI with other therapies is a logical and potentially promising approach, but it is too early to draw conclusions on specific combinations. As combinatorial therapies markedly increase the level of complexity, bedside-to-bench studies are warranted to gain deeper insight of underlying biological mechanisms which can be used to optimize future trials.
\end{abstract}

Keywords: Urothelial carcinoma; immunotherapy; chemotherapy; targeted therapy; antibody drug conjugate

Submitted Aug 18, 2020. Accepted for publication Jan 19, 2021.

doi: $10.21037 /$ tau-20-1177

View this article at: http://dx.doi.org/10.21037/tau-20-1177

\section{Introduction}

Radical cystectomy after neoadjuvant cisplatin-based chemotherapy is the standard therapy with curative intent for non-metastatic muscle-invasive bladder cancer (BC). Compared to other genitourinary cancers, the longterm survival rate is still poor for patients with advanced BC, mainly due to recurrences and metastatic spread (1). Before 2016, the recommended first-line therapy for locally advanced and metastatic urothelial carcinoma (mUC) was cisplatin-based chemotherapy, and there were no established, efficacious second-line options. Although $60-70 \%$ of eligible patients respond to cisplatin, long-term survival is only approximately $15 \%(2,3)$.

Around 40 years after the successful introduction of immunotherapy for non-muscle-invasive $\mathrm{BC}$ in the form of intravesical Bacillus-Chalmette-Guerin (BCG) instillation, several programmed death-1 (PD-1) and programmed death ligand-1 (PD-L1) immune checkpoint inhibitors (CPI) have been approved for the first- and second-line setting in mUC. Regulatory approval has been granted for 
the PD-1 inhibitors pembrolizumab and nivolumab and the PD-L1 inhibitors atezolizumab, avelumab and durvalumab in the United States, with more restricted approvals in other countries. Overall response rates (ORR) for CPI monotherapy range between $13 \%$ and $21 \%$ in unselected patient cohorts (4-11), and many of these responses are durable.

Undoubtedly, these CPI have revolutionized the treatment of mUC, with a subgroup of patients demonstrating sustained therapeutic responses. Nevertheless, progression and relapses occur in the majority of patients receiving CPI therapies. Intratumoral heterogeneity and the Darwinian selection of treatment-resistant tumor subclones, adaptive changes in tumor cells exposed to CPI, and alternative mechanisms of immune evasion have been proposed to be responsible for the failure of CPI monotherapy $(12,13)$. Rational combination of CPI with other therapies might overcome resistance by simultaneously utilizing different modes of action and target molecules. The comparatively low rate of severe adverse events with CPI and the approval of CPI combinations in renal cell carcinoma and melanoma have given impetus to clinical trials testing CPI combination therapy in $\mathrm{BC}(14,15)$. In this review, we will discuss available preliminary data and highlight important phase III trials for several CPI combinations in muscle-invasive, locally advanced and metastatic BC. We present the following article in accordance with the Narrative Review reporting checklist (available at http://dx.doi.org/10.21037/tau-20-1177).

\section{Dual checkpoint inhibition}

Neoadjuvant cisplatin-based chemotherapy followed by radical cystectomy demonstrated an absolute survival benefit of $5 \%$ to $8 \%$ compared to radical cystectomy alone (16). However, approximately half of patients is ineligible for cisplatin, due especially to impaired renal function and other co-morbidities. There are no alternative efficacious neoadjuvant therapies for this patient subpopulation.

The Phase Ib NABUCCO trial (NCT03387761) is investigating the combination of nivolumab and the CTLA4 (cytotoxic T-lymphocyte-associated Protein 4) inhibitor ipilimumab in the neoadjuvant setting in patients with stage III BC (cT3-4aN0M0 or $\geq \mathrm{cT} 1 \mathrm{~N}+\mathrm{M} 0$ ) ineligible or unwilling to receive cisplatin-based neoadjuvant chemotherapy (Table 1). Primary and secondary endpoints were feasibility and pathologic complete response (pCR) rate, respectively. In a preliminary report 23 of the 24 patients enrolled underwent radical cystectomy within
12 weeks from the first cycle and $54 \%$ of patients experienced high-grade adverse events (17). A pCR rate of $45 \%$ (10/22) was reported for the whole cohort, which was higher in PD$\mathrm{L} 1$ positive $(60 \%) v s$. negative patients $(22 \%)$. In comparison, the single arm phase II monotherapy trials with atezolizumab (ABACUS) and pembrolizumab (PURE-01) reported pCR rates of $31 \%$ and $37 \%$, respectively $(18,19)$.

The combination of the two CPI nivolumab and ipilimumab is also under investigation in the Phase I/II CheckMate 032 (NCT01928394) multicenter study after platinum-based chemotherapy for mUC (20). The three arms were NIVO3 (nivolumab $3 \mathrm{mg} / \mathrm{kg}$ monotherapy q2w), NIVO3+IPI1 (nivolumab $3 \mathrm{mg} / \mathrm{kg}$ plus ipilimumab $1 \mathrm{mg} /$ $\mathrm{kg}$ q3w for 4 cycles followed by nivolumab monotherapy $3 \mathrm{mg} / \mathrm{kg} \mathrm{q} 2 \mathrm{w}$ ) and NIVO1+IPI3 (nivolumab $1 \mathrm{mg} / \mathrm{kg}$ plus ipilimumab $3 \mathrm{mg} / \mathrm{kg} \mathrm{q} 3 \mathrm{w}$ for 4 cycles followed by nivolumab monotherapy $3 \mathrm{mg} / \mathrm{kg} \mathrm{q} 2 \mathrm{w}$ ). The primary endpoint was investigator-assessed ORR. Notably, the NIVO1+IPI3 arm was expanded with a protocol amendment and patients were included after the two other arms were closed. In an abstract report on this trial, 78, 104 and 92 patients were randomized into the NIVO3, NIVO3+IPI1 and NIVO1+IPI3 arms, respectively. In the NIVO3, NIVO3+IPI1 and NIVO1+IPI3 arms, the primary endpoint ORR was $25.6 \%$ (95\% CI: $16.4-36.8 \%$ ), $26.9 \%$ (95\% CI: $18.7-36.5 \%$ ) and $38.0 \%$ (95\% CI: $28.1-48.8 \%$ ). Notably, the extended follow-up data of the phase-III approval trial comparing pembrolizumab vs. chemotherapy as secondline treatment reported an ORR of $21.1 \%$ in the CPI arm, which is markedly lower compared to the ORR reported for the NIVO1+IPI3 arm in the CheckMate 032 study (4), as far as comparisons are possible across trials. Interestingly, in the NIVO1+IPI3 arm the ORR was $23.8 \%$ (95\% CI: $12.1-39.5 \%)$ in patients with low PD-L1 expressing tumors $(<1 \%)$ vs. $58.1 \%$ (95\% CI: 39.1-75.5\%) in patients with PD-L1 positive tumors. Treatment-related grade 3 and 4 adverse events occurred in 21 patients $(26.9 \%)$ of the NIVO3 arm, 32 patients (30.8\%) of the NIVO3+IPI1 arm and 36 patients $(39.1 \%)$ of the NIVO1+IPI3 arm, indicating that the higher dose ipilimumab may increase toxicity. There was one treatment-related death in both the NIVO3 and the NIVO3+IPI1 arms. It is important to note that this study was not powered to compare between each of the arms nor to compare the ORR between patients stratified by PD-L1 expression.

The promising results of the Phase I/II CheckMate 32 study were the foundation for the ongoing CheckMate 901 Phase III trial (NCT03036098) which is comparing 
Table 1 Clinical trials

\begin{tabular}{|c|c|c|c|c|c|c|}
\hline Name & Category & Compounds & Setting & Phase & NCT & Results \\
\hline BLASST-1 & $\mathrm{CPI}+$ Chemo & Nivolumab + chemo & NAC & II & NCT03294304 & Yes \\
\hline GU14-188 & $\mathrm{CPI}+$ Chemo & Pembrolizumab+ chemo & NAC & $\mathrm{lb} / \mathrm{ll}$ & NCT02365766 & Yes \\
\hline NABUCCO & $\mathrm{CPI}+\mathrm{CPI}$ & Nivolumab + ipilimumab & NAC & $\mathrm{lb}$ & NCT03387761 & Yes \\
\hline NIAGARA & $\mathrm{CPI}+$ Chemo & Durvalumab + chemo & Perioperative & III & NCT03732677 & No \\
\hline Keynote-866 & CPI + Chemo & Pembrolizumab+ chemo & Perioperative & III & NCT03924856 & No \\
\hline IMvigor 130 & $\mathrm{CPI}+$ Chemo & Atezolizumab + chemo & Metastasis, first-line & III & NCT02807636 & Yes \\
\hline $\begin{array}{l}\text { CheckMate } \\
901\end{array}$ & $\mathrm{CPI}+\mathrm{CPI} / \mathrm{CPI}+$ Chemo & $\begin{array}{l}\text { Nivolumab + ipilimumab/ } \\
\text { nivolumab + chemo }\end{array}$ & Metastasis, first-line & III & NCT03036098 & No \\
\hline Keynote-361 & $\mathrm{CPI}+$ Chemo & Pembrolizumab+ chemo & Metastasis, first-line & III & NCT02853305 & Yes \\
\hline PEANUT & $\mathrm{CPI}+$ Chemo & $\begin{array}{l}\text { Pembrolizumab+ albumin-bound } \\
\text { paclitaxel }\end{array}$ & Metastasis, first-line & II & NCT03464734 & Yes \\
\hline $\begin{array}{l}\text { CheckMate } \\
032\end{array}$ & $\mathrm{CPI}+\mathrm{CPI}$ & Nivolumab + ipilimumab & Metastasis, second-line & $\mathrm{I} / \mathrm{II}$ & NCT01928394 & Yes \\
\hline FIERCE22 & $\mathrm{CPI}+\mathrm{FGFR} 3$ & Pembrolizumab+ vofatamab & Metastasis, second-line & $\mathrm{lb} / \mathrm{II}$ & NCT03123055 & Yes \\
\hline EV-103 & $\begin{array}{l}\mathrm{CPI}+\text { Nectin } \\
\text { 4-inhibition }\end{array}$ & Pembrolizumab+ enfortumab-vedotin & Metastasis, first-line & $\mathrm{lb}$ & NCT0328854 & Yes \\
\hline
\end{tabular}

Chemo, chemotherapy; CPI, checkpoint inhibitor; FGFR3, fibroblast growth factor receptor 3.

nivolumab + ipilimumab (NIVO1+IPI3) vs. nivolumab + standard of care (SoC) chemotherapy vs. SoC chemotherapy alone in both cisplatin-eligible and -ineligible patients with mUC who have received no prior systemic chemotherapy (21). The results of this Phase III trial are eagerly awaited and will answer the question whether dual CPI combination is superior to CPI monotherapy or CPI in combination with chemotherapy.

In a trial that is similar in design to the CheckMate 901 trial, the phase III DANUBE study (NCT02516241) randomized more than 1,000 patients to treatment with the combination of durvalumab and the CTLA-4 inhibitor tremelimumab vs. durvalumab alone $v s$. SoC chemotherapy in patients with $\mathrm{mUC}$ in the first-line setting. Both cisplatin-eligible and -ineligible patients were included. The primary endpoint for durvalumab monotherapy $v s$.
SoC chemotherapy was overall survival (OS) in patients with PD-L1 positive tumors. The co-primary endpoint for durvalumab + tremelimumab vs. SoC chemotherapy was OS in patients regardless of tumor PD-L1 status. The DANUBE trail failed to meet either primary endpoint. In the PD-L1 positive cohort, there was no difference in OS between durvalumab monotherapy $v s$. SoC chemotherapy (HR 0.89, 0.71-1.11, P=0.3039). Furthermore, the combination of PD-L1 inhibition and CTLA4 inhibition did not outperform SoC chemotherapy (HR 0.85, 0.72 $1.02, \mathrm{P}=0.0751$ ) (22). The failure of this large trial once again underlines the obligation to conduct phase-III trials in order to validate results of earlier phase studies. This also reminds us that we have to be very cautious when predicting future directions based on early clinical trials. These lessons were reinforced with the results of the IMvigor 130 trial 
described below.

\section{Checkpoint-inhibitors combined with chemotherapy}

Cisplatin-based chemotherapy followed by CPI is the backbone of systemic therapy in mUC. Since both therapies have very different mechanisms of action, and since chemotherapy can potentially increase antigen presentation from tumors that could augment the effect of CPI, combining both therapies have been a logical next step in clinical trial design, with the anticipation that this might increase the number of patients who are able to achieve long-term disease control.

The phase II BLASST-1 (Bladder Cancer Signal Seeking Trial) study (NCT03294304) investigated neoadjuvant nivolumab together with the SoC gemcitabine and cisplatin in patients with muscle-invasive $\mathrm{BC}(\mathrm{cT} 2-\mathrm{T} 4 \mathrm{a}, \mathrm{N} \leq 1$, $\mathrm{M} 0)$. Primary and secondary endpoints were pathological response ( $\leq$ ypT1, N0) and safety as well as progressionfree survival (PFS), respectively. The combination of CPI and chemotherapy was administered for 4 cycles followed by radical cystectomy within 8 weeks after the last systemic therapy. In a preliminary report based on all 41 patients enrolled, the primary endpoint was reached in $65.8 \%(27 / 41)(23)$. Grade 3 or 4 adverse events were observed in $20 \%$ of the patients and were largely attributed to the chemotherapy component. Neither delay of radical cystectomy nor unexpected surgical complications were observed. The preliminary results of this study are noteworthy, as the pathological response rate was higher than historical controls treated with neoadjuvant gemcitabine and cisplatin (24), although the difference in response rates to neoadjuvant MVAC is not vastly different (38.5\% ypT0N0, down-staging rate not available) (25), and randomized trials will be necessary to show true superiority.

Building on the promising signal of the BLASST-1 trial, the ENERGIZE phase III study (NCT03661320) has been launched to investigate the combination of cisplatinbased neoadjuvant chemotherapy and nivolumab with or without the selective IDO1 (indoleamine 2,3-dioxygenase) inhibitor linrodostat mesylate (BMS-986205) (26). In this trial the CPI +/- IDO1 inhibitor therapy will continue postoperatively. This is one of the first trials to introduce triple therapy combinations including chemotherapy and CPI, and it suggests that triplet combinations with other targeted therapies are likely on the horizon.

Similar to BLASST-1, the single-arm phase II SAKK
06/17 trial (NCT03406650) investigated the combination of durvalumab and gemcitabine/cisplatin in the perioperative setting (27). An interim analysis revealed that 9 of 34 (26.5\%) patients had pCR and $15(44.1 \%)$ had ypT $\leq 1 \mathrm{~N} 0$. Four patients who did not proceed to cystectomy were considered non-responders, which may artificially reduce apparent efficacy.

The GU14-188 trial (NCT02365766) is a third phase $\mathrm{Ib} / \mathrm{II}$ trial investigating the combination of chemotherapy and CPI in the neoadjuvant setting in patients with cT24aN0M0 BC (28). Cohort 1 included cisplatin-eligible patients receiving 4 cycles of cisplatin, gemcitabine and pembrolizumab and cohort 2 included cisplatinineligible patients receiving 3 cycles of gemcitabine and pembrolizumab (29). Of 43 patients enrolled in cohort 1 , $61.1 \%$ (95\% CI: $0.45,0.75)$ were down-staged to yp $\mathrm{T} \leq 1 \mathrm{~N} 0$ and $44.4 \%$ had a complete pathological response (ypT0N0). In cohort $2,51.6 \%$ (95\% CI: $0.35,0.68)$ were yp $\mathrm{T} \leq 1 \mathrm{~N} 0$ and $45.2 \%$ were ypT0N0 at radical cystectomy. These response rates are particularly encouraging in cisplatinineligible patients who otherwise do not have options for neoadjuvant therapy, and they are higher than response rates reported for neoadjuvant CPI monotherapy $(18,19)$. PD-L1 expression did not correlate with outcome in the GU14-188 study.

Based on these promising results, the phase III KEYNOTE-866 study (NCT03924856) is investigating perioperative pembrolizumab + chemotherapy $v s$. perioperative placebo + chemotherapy (27). Pembrolizumab will be continued for up to 13 -week cycles after surgery. Patients with non-metastatic muscle-invasive $\mathrm{BC}$ (cT2-4aN0M0) are being enrolled. The co-primary endpoints will be $\mathrm{pCR}$ in all patients and in patients with PD-L1 positive tumors. OS, toxicity and pathological down-staging are secondary endpoints. The NIAGARA trial (NCT03732677) is a similar large open-label phase III trial in the same disease state that will randomize patients to neoadjuvant gemcitabine/cisplatin + perioperative durvalumab versus neoadjuvant gemcitabine/ cisplatin alone (30).

In the metastatic setting, the IMvigor 130 trial (NCT02807636) was the first phase III trial to report results from combined CPI and chemotherapy (11). This study included both cisplatin-eligible and -ineligible patients in the first-line. Patients were randomized into three arms: atezolizumab plus platinum-based chemotherapy (group A), atezolizumab monotherapy (group B) or placebo plus platinum-based chemotherapy (group C). The co-primary endpoints were PFS and OS comparing only group A 
versus group $\mathrm{C}$, with no comparisons planned with group B. The final PFS analysis and an interim OS analysis have been reported, with the final OS results pending. The trial enrolled 1,213 patients and the median follow-up was 11.8 months (IQR, 6.1-17.2). PFS was 8.2 months (95\% CI: 6.5-8.3) in group A and 6.3 months (95\% CI: 6.2-7.0) in group C (HR: 0.82, 95\% CI: 0.70-0.96; $\mathrm{P}=0.007$ ), indicating superiority of the combination over chemotherapy alone. Interim OS was 16.0 months (13.9-18.9) in group A and 13.4 months (12.0-15.2) in group C (HR: 0.83, $95 \%$ CI: $0.69-1.00, \mathrm{P}=0.027)$ and therefore did not reach the prespecified level of significance $(\mathrm{P}=0.007)$. Grade 3 and 4 adverse events were seen in $81 \%, 15 \%$ and $81 \%$ for group A, B and C, respectively. The ORR was similar between group A and group C (47\% vs. 44\%) although the rate of complete responses was greater in the combination arm (13\% vs. $7 \%$ ). Taken together, this study shows promising results for the combination of chemotherapy and CPI, but the final OS analysis will determine the true added value of combination therapy, since patients progressing on platinum-based chemotherapy in group $\mathrm{C}$ still have the option of receiving CPI as a second-line therapy, which could impact OS.

Two other large phase III trials, similar to IMvigor130, have investigated the combination of chemotherapy and CPI for first-line treatment of mUC (31). The NILE study (NCT03682068) has not yet completed accrual, and plans to randomize 1434 patients to durvalumab + SoC chemotherapy versus durvalumab + tremelimumab + SoC chemotherapy versus $\mathrm{SoC}$ chemotherapy alone (31). The KEYNOTE-361 trial (NCT02853305) has completed accrual, randomizing 1,010 patients to pembrolizumab + SoC chemotherapy versus pembrolizumab monotherapy versus SoC chemotherapy alone. Co-primary endpoints were PFS and OS in the intention to treat population. Initial results were presented at ESMO Virtual Congress 2020 (32). The prespecified level of statistical significance for both primary endpoints, PFS (HR 0.78, 9.65-0.93, $\mathrm{P}=0.0033)$ and $\mathrm{OS}(\mathrm{P}=0.86,0.72-1.02, \mathrm{P}=0.0407)$, were not reached.

The modest results of IMvigor130 and the reported failure of KEYNOTE-361 to meet primary endpoints have tempered the enthusiasm for combined CPI and chemotherapy for first-line therapy of mUC, especially in light of the more pronounced OS benefit of switch maintenance avelumab after first-line platinum-chemotherapy in the JAVELIN Bladder 100 trial (NCT02603432) (33). It is possible that the same tumors respond to both CPI and chemotherapy, so that the additive benefit is modest. Definitive conclusions cannot be drawn until complete trial results have been reported.

One phase II trial tested CPI plus chemo for second-line treatment of mUC. The PEANUT trial (NCT03464734) investigated the combination of pembrolizumab and nanoparticle albumin-bound paclitaxel (34). Seventy patients were enrolled and an encouraging ORR of $39 \%$ and CR of $14.3 \%$ were reported.

\section{Other combinatorial approaches including checkpoint-inhibitors}

Combining CPI with other compounds is not limited to adding chemotherapy or another CPI. Two other drugs have been approved by the FDA for second- and thirdline treatment of patients with mUC, and both are being tested in combination with CPI. The pan-FGFR inhibitor erdafitinib has demonstrated efficacy in a single arm phase II study for patients with specific alterations in FGFR2 and FGFR3 (35). In the NORSE trial (NCT03473743) combining erdafitinib plus cetrelimab, a newer PD-1 inhibitor, 5 of 7 patients (71\%) demonstrated an objective response (36). This combination is now being tested in a randomized phase II trial for first-line management of cisplatin-ineligible patients with mUC (NCT03473743). In the second-line, post-platinum mUC setting, the Phase-Ib/ II FIERCE22 trial (NCT03123055) tested the combination of pembrolizumab and a selective anti-FGFR3-antibody (vofatamab). This trial showed promising results on interim analysis with an ORR of $29.6 \%$ (37).

The antibody-drug conjugate enfortumab-vedotin targeting Nectin-4 was granted accelerated FDA approval based on the single arm phase II EV-201 trial, which showed an ORR of $44 \%$ including a CR of $12 \%$ in mUC patients previously treated with both platinum-based chemotherapy and CPI (38). Early results from the phase Ib EV-103 study (NCT03288545) testing the combination of pembrolizumab and enfortumab-vedotin for first-line treatment of 45 cisplatin-ineligible mUC patients demonstrated an ORR of $73.3 \%$ including a CR of $15.6 \%$, and a disease-control rate of $93.3 \%$, which is extraordinarily high compared to other compounds (39). EV-103 has sparked great interest in the corresponding phase III trial EV-302 (NCT04223856) which is randomizing patients with $\mathrm{mUC}$ to enfortumab plus pembrolizumab with or without platinum-based chemotherapy, versus chemotherapy alone.

Other drugs under investigation in combination with 
CPI are PARP-inhibitors (NCT03459846), interleukin-2 agonists (NCT04209114) and multiple-kinase inhibitors (NCT03898180), among many others novel agents.

\section{Conclusions}

Although CPI monotherapy leads to durable responses in a subgroup of patients, most patients with locally advanced and mUC still do not benefit. Progression under CPI is the rule, and further improvement of patient outcomes will likely depend on effective combination therapies that build on the success of CPI by simultaneously blocking independent oncologic pathways. Consequentially, there is much anticipation around the many clinical trials investigating these combinations, especially the large phase III studies that may be practice-changing. Most trials to date have published either no results or only preliminary results, and there have been some reports and press releases indicating that large phase III trials in the first-line mUC setting have failed to meet the targeted endpoints. It is therefore impossible currently to project the future role of CPI combined with other drugs without awaiting the results of the relevant trials. As the level of complexity increases exponentially with combinatorial therapies, a deeper understanding of underlying biologic mechanisms through bedside-to-bench studies is warranted, in addition to additional clinical trials. This concentrated effort should promote better outcomes for patients with urothelial carcinoma.

\section{Acknowledgments}

Funding: None.

\section{Footnote}

Provenance and Peer Review: This article was commissioned by the Guest Editor (Tilman Todenhöfer) for the series "Management of Advanced Genitourinary Malignancies" published in Translational Andrology and Urology. The article has undergone external peer review.

Reporting Checklist: The authors have completed the Narrative Review reporting checklist. Available at http:// dx.doi.org/10.21037/tau-20-1177

Conflicts of Interest: Both authors have completed the ICMJE uniform disclosure form (available at http://dx.doi. org/10.21037/tau-20-1177). The series "Management of Advanced Genitourinary Malignancies" was commissioned by the editorial office without any funding or sponsorship. Dr. GBS reports to have interpersonal conflict of interest: Merck and received (travel) grants and/or an honorarium from the following commercial organizations within the last three years: Roche, Merck, Ipsen, Astellas. He is currently participating in or have participated in clinical trials sponsored by the following pharmaceutical companies: Merck, BMS, AstraZeneca. Dr. VCB reports other from Janssen, other from Merck, other from Roche/Genentech, other from BMS, other from Urogen, other from Asieris, other from EMD Serono, other from Bayer, other from TerSera, other from Astellas, other from AbbVie, other from AstraZeneca, other from Ferring, other from Fergene, other from H3-Biomedicine, other from Sanofi, other from Biosyent, other from Pfizer, grants from iProgen, nonfinancial support from Decipher Biosciences, outside the submitted work. The authors have no other conflicts of interest to declare.

Ethical Statement: The authors are accountable for all aspects of the work in ensuring that questions related to the accuracy or integrity of any part of the work are appropriately investigated and resolved.

Open Access Statement: This is an Open Access article distributed in accordance with the Creative Commons Attribution-NonCommercial-NoDerivs 4.0 International License (CC BY-NC-ND 4.0), which permits the noncommercial replication and distribution of the article with the strict proviso that no changes or edits are made and the original work is properly cited (including links to both the formal publication through the relevant DOI and the license). See: https://creativecommons.org/licenses/by-nc-nd/4.0/.

\section{References}

1. Hautmann RE, de Petriconi RC, Pfeiffer C, et al. Radical cystectomy for urothelial carcinoma of the bladder without neoadjuvant or adjuvant therapy: long-term results in 1100 patients. Eur Urol 2012;61:1039-47.

2. Sternberg CN, de Mulder PH, Schornagel JH, et al. Randomized phase III trial of high-dose-intensity methotrexate, vinblastine, doxorubicin, and cisplatin (MVAC) chemotherapy and recombinant human granulocyte colony-stimulating factor versus classic MVAC in advanced urothelial tract tumors: European 
Organization for Research and Treatment of Cancer Protocol no. 30924. J Clin Oncol 2001;19:2638-46.

3. von der Maase H, Sengelov L, Roberts JT, et al. Longterm survival results of a randomized trial comparing gemcitabine plus cisplatin, with methotrexate, vinblastine, doxorubicin, plus cisplatin in patients with bladder cancer. J Clin Oncol 2005;23:4602-8.

4. Necchi A, Fradet Y, Bellmunt J, et al. Three-year follow-up from the phase III KEYNOTE-045 trial: Pembrolizumab (Pembro) versus investigator\&\#x2019; s choice (paclitaxel, docetaxel, or vinflunine) in recurrent, advanced urothelial cancer (UC). Ann Oncol 2019;30:v366-7.

5. Powles T. Atezolizumab versus chemotherapy in patients with platinum-treated locally advanced or metastatic urothelial carcinoma (IMvigor211): a multicentre, open-label, phase 3 randomised controlled trial. Lancet 2018;391:748-57.

6. Powles T, O'Donnell PH, Massard C, et al. Updated efficacy and tolerability of durvalumab in locally advanced or metastatic urothelial carcinoma. J Clin Oncol 2017;35:286.

7. Patel MR, Ellerton J, Infante JR, et al. Avelumab in metastatic urothelial carcinoma after platinum failure (JAVELIN Solid Tumor): pooled results from two expansion cohorts of an open-label, phase 1 trial. Lancet Oncol 2018;19:51-64.

8. Sharma P, Retz M, Siefker-Radtke A, et al. Nivolumab in metastatic urothelial carcinoma after platinum therapy (CheckMate 275): a multicentre, single-arm, phase 2 trial. Lancet Oncol 2017;18:312-22.

9. Balar AV, Castellano D, O'Donnell PH, et al. First-line pembrolizumab in cisplatin-ineligible patients with locally advanced and unresectable or metastatic urothelial cancer (KEYNOTE-052): a multicentre, single-arm, phase 2 study. Lancet Oncol 2017;18:1483-92.

10. Balar AV, Galsky MD, Rosenberg JE, et al. Atezolizumab as first-line treatment in cisplatin-ineligible patients with locally advanced and metastatic urothelial carcinoma: a single-arm, multicentre, phase 2 trial. Lancet 2017;389:67-76.

11. Galsky MD, Arija JÁA, Bamias A, et al. Atezolizumab with or without chemotherapy in metastatic urothelial cancer (IMvigor130): a multicentre, randomised, placebocontrolled phase 3 trial. Lancet 2020;395:1547-57.

12. Gerlinger M, Catto JW, Orntoft TF, et al. Intratumour heterogeneity in urologic cancers: from molecular evidence to clinical implications. Eur Urol 2015;67:729-37.

13. Warrick JI, Sjodahl G, Kaag M, et al. Intratumoral
Heterogeneity of Bladder Cancer by Molecular Subtypes and Histologic Variants. Eur Urol 2019;75:18-22.

14. Motzer RJ, Tannir NM, McDermott DF, et al. Nivolumab plus Ipilimumab versus Sunitinib in Advanced Renal-Cell Carcinoma. N Engl J Med 2018;378:1277-90.

15. Wolchok JD, Chiarion-Sileni V, Gonzalez R, et al. Overall Survival with Combined Nivolumab and Ipilimumab in Advanced Melanoma. N Engl J Med 2017;377:1345-56.

16. Advanced Bladder Cancer (ABC) Meta-analysis Collaboration. Neoadjuvant chemotherapy in invasive bladder cancer: update of a systematic review and metaanalysis of individual patient data advanced bladder cancer (ABC) meta-analysis collaboration. Eur Urol 2005;48:2025; discussion 205-6.

17. Van der Heijden MS, van Dijk N, Smit L, et al. Preoperative ipilimumab and nivolumab in locoregionally advanced, stage III, urothelial cancer (NABUCCO). Ann Oncol 2019;30:v358.

18. Necchi A, Raggi D, Gallina A, et al. Updated Results of PURE-01 with Preliminary Activity of Neoadjuvant Pembrolizumab in Patients with Muscle-invasive Bladder Carcinoma with Variant Histologies. Eur Urol 2020;77:439-46.

19. Powles T, Kockx M, Rodriguez-Vida A, et al. Clinical efficacy and biomarker analysis of neoadjuvant atezolizumab in operable urothelial carcinoma in the ABACUS trial. Nat Med 2019;25:1706-14.

20. Sharma P, Siefker-Radtke A, Braud Fd, et al. Nivolumab Alone and With Ipilimumab in Previously Treated Metastatic Urothelial Carcinoma: CheckMate 032 Nivolumab $1 \mathrm{mg} / \mathrm{kg}$ Plus Ipilimumab $3 \mathrm{mg} / \mathrm{kg}$ Expansion Cohort Results. J Clin Oncol 2019;37:1608-16.

21. Galsky MD, Powles T, Li S, et al. A phase 3, openlabel, randomized study of nivolumab plus ipilimumab or standard of care (SOC) versus SOC alone in patients (pts) with previously untreated unresectable or metastatic urothelial carcinoma (mUC; CheckMate 901). J Clin Oncol 2018;36:TPS539.

22. Powles T, van der Heijden MS, Castellano D, et al. Durvalumab alone and durvalumab plus tremelimumab versus chemotherapy in previously untreated patients with unresectable, locally advanced or metastatic urothelial carcinoma (DANUBE): a randomised, open-label, multicentre, phase 3 trial. Lancet Oncol 2020;21:1574-88.

23. Gupta S, Sonpavde G, Weight CJ, et al. Results from BLASST-1 (Bladder Cancer Signal Seeking Trial) of nivolumab, gemcitabine, and cisplatin in muscle invasive bladder cancer (MIBC) undergoing cystectomy. J Clin 
Oncol 2020;38:439.

24. Yuh BE, Ruel N, Wilson TG, et al. Pooled Analysis of Clinical Outcomes with Neoadjuvant Cisplatin and Gemcitabine Chemotherapy for Muscle Invasive Bladder Cancer. J Urol 2013;189:1682-6.

25. Grossman HB, Natale RB, Tangen CM, et al. Neoadjuvant chemotherapy plus cystectomy compared with cystectomy alone for locally advanced bladder cancer. N Engl J Med 2003;349:859-66.

26. Sonpavde G, Necchi A, Gupta S, et al. A phase 3 randomized study of neoadjuvant chemotherapy (NAC) alone or in combination with nivolumab (NIVO) \pm BMS986205 in cisplatin-eligible muscle invasive bladder cancer (MIBC). J Clin Oncol 2019;37:TPS4587.

27. Cathomas R, Petrausch U, Hayoz S, et al. Perioperative chemoimmunotherapy with durvalumab (Durva) in combination with cisplatin/gemcitabine (Cis/Gem) for operable muscle-invasive urothelial carcinoma (MIUC): Preplanned interim analysis of a single-arm phase II trial (SAKK 06/17). J Clin Oncol 2020;38:499.

28. Hoimes CJ, Adra N, Fleming MT, et al. Phase Ib/II neoadjuvant (N-) pembrolizumab (P) and chemotherapy for locally advanced urothelial cancer (laUC): Final results from the cisplatin (C)- eligible cohort of HCRN GU14188. J Clin Oncol 2020;38:5047.

29. Kaimakliotis HZ, Adra N, Kelly WK, et al. Phase II neoadjuvant (N-) gemcitabine $(\mathrm{G})$ and pembrolizumab (P) for locally advanced urothelial cancer (laUC): Interim results from the cisplatin (C)-ineligible cohort of GU14188. J Clin Oncol 2020;38:5019.

30. Powles T, Meeks JJ, Galsky MD, et al. A phase III, randomized, open label, multicenter, global study of efficacy and safety of durvalumab in combination with gemcitabine+cisplatin $(\mathrm{G}+\mathrm{C})$ for neoadjuvant treatment followed by durvalumab alone for adjuvant treatment in muscle-invasive bladder cancer (MIBC) (NIAGARA). J Clin Oncol 2019;37:TPS4592.

31. Galsky MD, Necchi A, Sridhar SS, et al. A phase III, randomized, open-label, multicenter, global study of firstline (1L) durvalumab in combination with standard of care (SoC) chemotherapy and durvalumab in combination with tremelimumab and soc chemotherapy versus soc chemotherapy alone in patients with unresectable locally advanced or metastatic urothelial cancer (UC). J Clin Oncol 2019;37:TPS499.

32. Alva A, Csőszi T, Ozguroglu M, et al. LBA23

Pembrolizumab (P) combined with chemotherapy (C) vs $\mathrm{C}$ alone as first-line (1L) therapy for advanced urothelial carcinoma (UC): KEYNOTE-361. Ann Oncol 2020;31:S1155.

33. Powles T, Park SH, Voog E, et al. Avelumab Maintenance Therapy for Advanced or Metastatic Urothelial Carcinoma. N Engl J Med 2020;383:1218-30.

34. Giannatempo P, Calareso G, Bandini M, et al. Final results of PEANUT: Pembrolizumab and nanoparticle albuminbound paclitaxel (nab-paclitaxel) as salvage therapy for metastatic urothelial carcinoma (UC). J Clin Oncol 2020;38:5017.

35. Loriot Y, Necchi A, Park SH, et al. Erdafitinib in Locally Advanced or Metastatic Urothelial Carcinoma. N Engl J Med 2019;381:338-48.

36. Moreno V, Loriot Y, Valderrama BP, et al. Does escalation results from phase $\mathrm{Ib} / \mathrm{II}$ Norse study of erdafitinib (ERDA) + PD-1 inhibitor JNJ-63723283 (Cetrelimab [CET]) in patients (pts) with metastatic or locally advanced urothelial carcinoma (mUC) and selected fibroblast growth factor receptor (FGFR) gene alterations. J Clin Oncol 2020;38:511.

37. Siefker-Radtke AO, Lugowska I, Tupikowski K, et al. Clinical activity of vofatamab (V), an FGFR3 selective antibody in combination with pembrolizumab $(\mathrm{P})$ in metastatic urothelial carcinoma (mUC), updated interim analysis of FIERCE-22. Ann Oncol 2019;30:v365.

38. Rosenberg JE, O'Donnell PH, Balar AV, et al. Pivotal Trial of Enfortumab Vedotin in Urothelial Carcinoma After Platinum and Anti-Programmed Death 1/Programmed Death Ligand 1 Therapy. J Clin Oncol 2019;37:2592-600.

39. Rosenberg JE, editor. Study EV-103: Preliminary durability results of enfortumab vedotin plus pembrolizumab for locally advanced or metastatic urothelial carcinoma 2020; Genitourinary Cancers Symposium: American Society of Clinical Oncology.
Cite this article as: Schulz GB, Black PC. Combination therapies involving checkpoint-inhibitors for treatment of urothelial carcinoma: a narrative review. Transl Androl Urol 2021;10(10):4014-4021. doi: 10.21037/tau-20-1177 